\title{
Building a strong foundation
}

\author{
Authors: Kieran Palmer, George Sismey, Shivani Gor, James Lambert, Lauren King, Mehak Chadha, Kathryn Killicoat \\ and Billy Cheung*
}

\section{Introduction}

Final year medical students often feel apprehensive and anxious when transitioning from student to doctor. Studies have shown that increased length of shadowing and specific 'preparing for practice' teaching improve confidence. We targeted this transition by delivering an extensive medical education preparation programme. We aimed to improve student preparation and ability, with a view to improving patient safety.

\section{Materials and methods}

We designed a comprehensive programme to target history, examination and clinical skills, while providing experience of being on call. There were four streams:

$>$ focused bedside teaching

$>$ an 'on-call hour' simulation exercise

> a comprehensive 11 station formative objective structured clinical examination (OSCE) examination

> 'Thriving and Surviving' lecture series, incorporating the new national NEWS2 scoring system for unwell patients.

Questionnaires were completed before and after the course. We used a Likert scale for various feedback components, which included items from the General Medical Council Outcomes for Graduates specification. ${ }^{1}$

\section{Results and discussion}

Quantitative and qualitative feedback was collected during each part of the teaching programme. The on-call simulation questionnaires assessed candidates' confidence in answering bleeps, prioritising tasks, handing over and escalation. Our results demonstrated a significant increase in mean confidence and perceived abilities in all areas assessed (38\% to $94 \%$, $\mathrm{p}<0.001)$. Students also felt that it identified and addressed many points which were otherwise absent from the standard medical curriculum. Overwhelmingly positive feedback from the OSCE and bedside teaching indicated that students appreciated appropriately pitched, structured teaching as preparation for their final exams and clinical practice.

Authors: Homerton University Hospital NHS Foundation Trust ${ }^{*} \mathrm{RCP}$ chief registrar

\section{Conclusion}

Our feedback further confirms that students often feel uncertain and anxious when starting work. Our results suggest that a targeted 'on-the-job' teaching course improves confidence for clinical practice across all domains. We recommend similar implementation across different medical schools in the future.

\section{Reference}

1 General Medical Council. Outcomes for graduates. London: GMC, 2018. 